\title{
Total Sample Conditioning and Preparation of Nanoliter Volumes for Electron Microscopy
}

\author{
Stefan A. Arnold, ${ }^{\dagger, \dagger}$ Stefan Albiez, ${ }^{\dagger}$ Nadia Opara, ${ }^{\dagger, \dagger, \S}$ Mohamed Chami, ${ }^{\dagger}$ Claudio Schmidli, ${ }^{\dagger, \dagger}$
} Andrej Bieri, ${ }^{\dagger}$ Celestino Padeste, ${ }^{\S}$ Henning Stahlberg, ${ }^{\dagger}$ and Thomas Braun ${ }^{*}{ }^{\dagger}$

${ }^{\dagger}$ Center for Cellular Imaging and NanoAnalytics (C-CINA), Biozentrum and ${ }^{\ddagger}$ Swiss Nanoscience Institute, University of Basel, 4001 Basel, Switzerland

${ }^{\S}$ Paul Scherrer Institute (PSI), 5232 Villigen, Switzerland

Supporting Information

\begin{abstract}
Electron microscopy (EM) entered a new era with the emergence of direct electron detectors and new nanocrystal electron diffraction methods. However, sample preparation techniques have not progressed and still suffer from extensive blotting steps leading to a massive loss of sample. Here, we present a simple but versatile method for the almost lossless sample conditioning and preparation of nanoliter volumes of biological samples for EM, keeping the sample under close to physiological condition. A microcapillary is used to aspirate $3-5 \mathrm{~nL}$ of sample. The microcapillary tip is immersed into a reservoir of negative

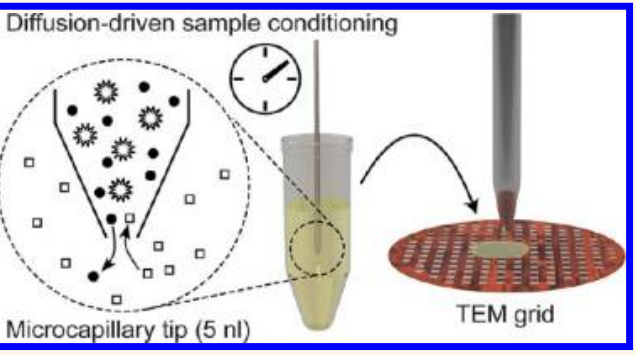
stain or trehalose, where the sample becomes conditioned by diffusive exchange of salt and heavy metal ions or sugar molecules, respectively, before it is deposited as a small spot onto an EM grid. We demonstrate the use of the method to prepare protein particles for imaging by transmission EM and nanocrystals for analysis by electron diffraction. Furthermore, the minute sample volume required for this method enables alternative strategies for biological experiments, such as the analysis of the content of a single cell by visual proteomics, fully exploiting the single molecule detection limit of EM.
\end{abstract}

KEYWORDS: electron microscopy, negative staining, single-cell analysis, visual proteomics, nanocrystal, electron diffraction

I $\mathrm{n}$ recent years, electron microscopy (EM) has experienced a renaissance in the field of biological research. This is based on improved instrumentation, through direct electron detection cameras, ${ }^{1-3}$ and advances in image processing software systems. ${ }^{4,5}$ Related fields such as electron diffraction of $3 \mathrm{D}$ nanocrystals has equally progressed significantly. ${ }^{6,7}$ While cryoEM of two-dimensional crystals ${ }^{8}$ or single protein "particles" allows the structural investigation of biological samples under physiological conditions at high resolution, the enhanced signalto-noise ratio (SNR) makes negative stain $\mathrm{EM}^{10}$ especially suited to the analysis of complex samples, e.g., dynamic protein assemblies.

Unfortunately, standard methods used to prepare samples for EM suffer from two major drawbacks: Typically, a large sample volume (around $3 \mu \mathrm{L}$ ) containing the biological species at high concentration $(0.1 \mathrm{mg} / \mathrm{mL}$ to $3 \mathrm{mg} / \mathrm{mL}$ protein $)$ is adsorbed to a carbon-film coated EM grid, blotted with filter paper, stained, and blotted again, before being air-dried for inspection in a transmission electron microscope (TEM). These blotting steps remove more than $99 \%$ of the sample volume. In addition, since adsorption to the carbon film depends on the specific samplecarbon interaction, blotting can promote the uncontrolled and preferential removal of sample subclasses, leading to a nonrepresentative sample population on the EM grid, which can interfere with the analysis of (large) biomolecule assemblies, such as proteoliposomes, dynamic protein complexes, or other (synthetic) particles. The effect is even more pronounced when protocols include washing procedures.

Advanced methods for the preparation of EM samples are needed. Recent efforts have mainly addressed three aspects: (i) the reduction of sample loss, (ii) the increase of throughput, and (iii) the reduction of sample consumption. Wash- and blottingfree negative staining procedures were recently developed to reduce sample loss, i.e., biological samples can now be stained in microdialysis fibers and directly deposited onto an EM grid in a controlled manner. ${ }^{11}$ However, as some sample is still lost along the microcapillary and dialysis fiber of the inline staining system, that method does not allow the analysis of nanoliter sample volumes or solutions with low protein concentrations. To increase throughput, multiple grids can be prepared in parallel ${ }^{12}$ or printing ${ }^{13}$ and inkjet picoliter dispensing tools such as the Spotiton system ${ }^{14}$ can be used to deposit an array of samples on a single EM grid. Although, these are exciting developments and represent an excellent method to prepare minimal volumes of

Received: February 22, 2016

Accepted: April 13, 2016

Published: April 13, 2016 

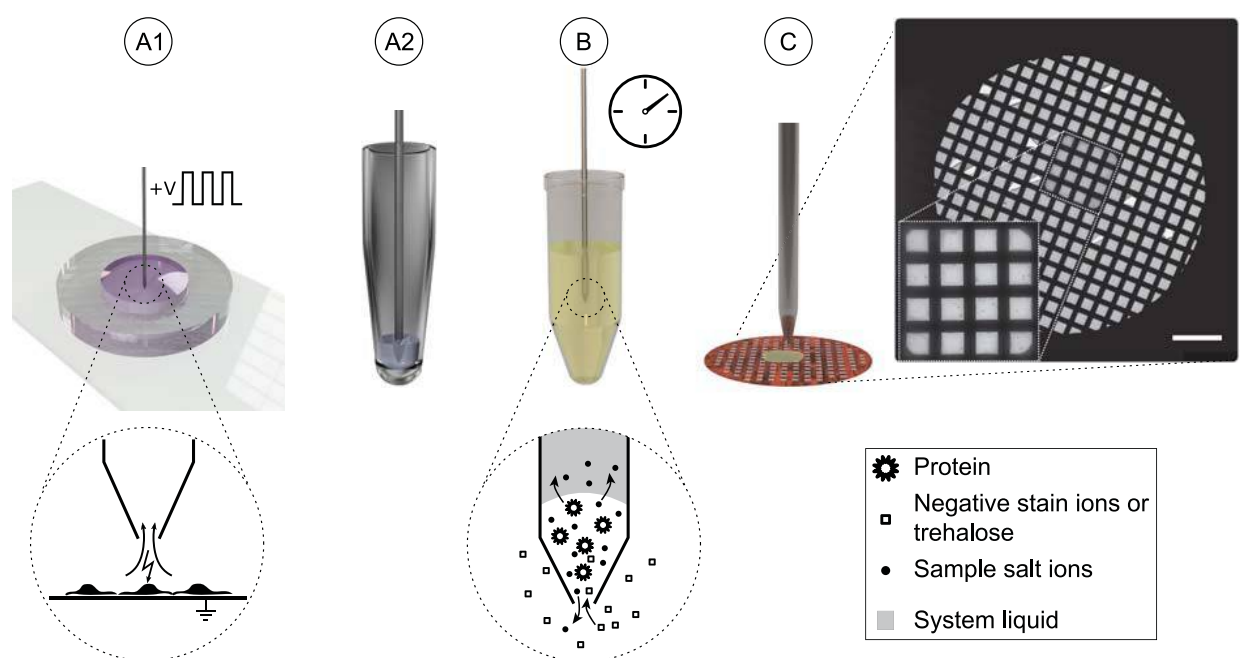

Figure 1. Sample preparation process. (A1) Electroporation and aspiration (lysis) of a single cell from cell culture; or (A2) aspiration of $5 \mathrm{~nL}$ of sample from sample stock. (B) Immersion of loaded microcapillary tip into the conditioning liquid, here negative stain, for diffusion-driven desalting and staining of the sample. (C) Deposition of $5 \mathrm{~nL}$ of sample on a glow-discharged EM grid and analysis by EM. Scale bar $500 \mu \mathrm{m}$.

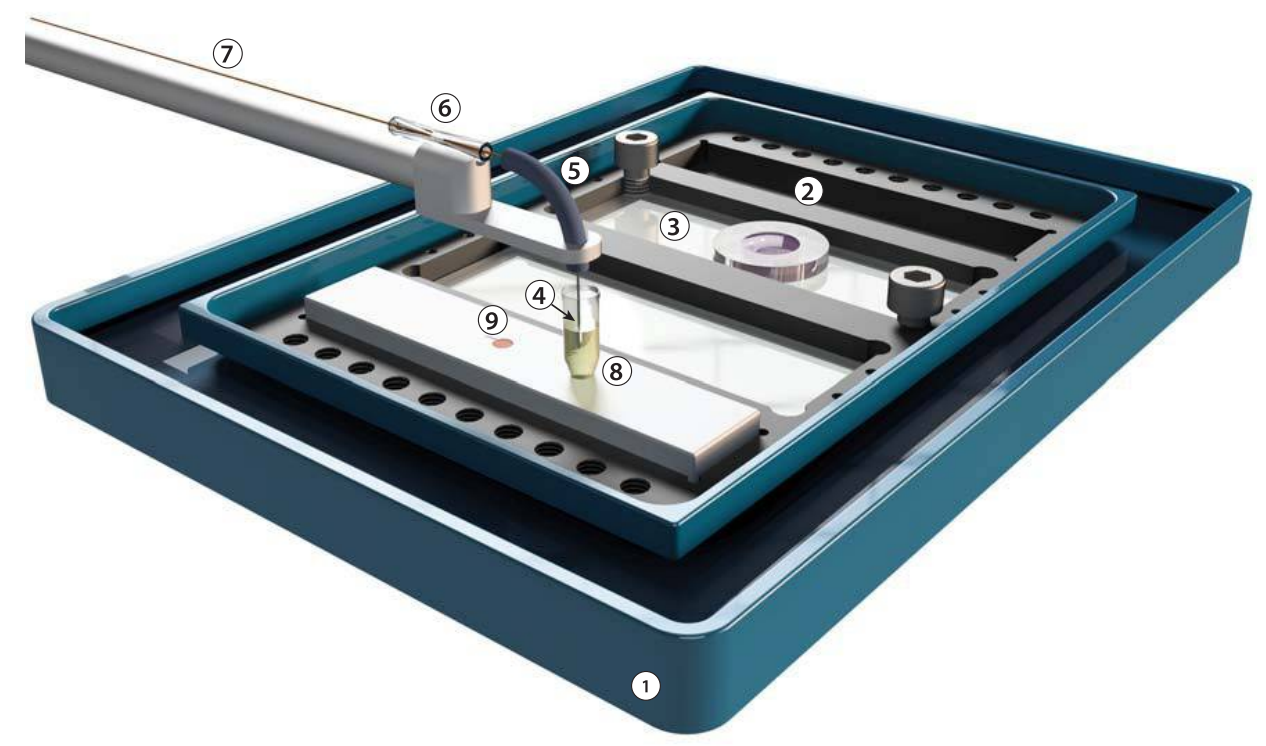

Figure 2. Single-cell lysis and sample conditioning setup. The essential components are placed on a work platform mounted in a live-cell incubator stage (1). For single-cell experiments, miniaturized Petri dishes are used to culture the cells (2). These poly(dimethylsiloxane) (PDMS)-based wells are reversibly bonded to an indium tin oxide (ITO)-coated microscopy slide (3). Cells are lysed by electroporation, and the lysate is immediately aspirated $\left(3-5 \mathrm{~nL}\right.$ ) by a microcapillary $(4) .{ }^{13}$ Alternatively, samples can be loaded into the capillary from a sample well (not shown). The microcapillary is guided by a conductive sleeve (5) and connected by a press-fit mechanism (6) and a microcapillary (7) to a highprecision pump system (not shown). All capillaries are filled with system liquid (water), which is in direct contact with the aspirated sample (see Figure 1). For sample conditioning, the microcapillary tip is immersed in negative stain or trehalose solution contained in a $200 \mu \mathrm{L}$ tube $(8)$. Subsequently, the sample is deposited on a carbon-coated EM grid held in place by a groove (9) or a pair of tweezers (not shown).

sample per grid in a fast and efficient manner, these approaches rely on a larger sample stock, and for preparing negative staining TEM grids, they would require additional washing and/or blotting steps.

Here, we present a method for negative stain sample preparation or trehalose embedding ${ }^{15}$ that only consumes $5 \mathrm{~nL}$ or less of biological material. For sample conditioning and EM grid preparation, a microcapillary with sample loaded in its tip is immersed into a reservoir of conditioning solution; the conditioning is driven by diffusion, utilizing the different diffusion constants of salt molecules and proteins (Figure 1). We show that this method has manifold applications, e.g., to prepare single particles for cryo- or negative stain imaging, single cells for visual proteomics, ${ }^{11,16}$ or nanocrystals for electron diffraction studies.

\section{RESULTS AND DISCUSSION}

We implemented the sample preparation method (Figure 1) in an instrument initially developed for single-cell lysis. ${ }^{16}$ The setup (Figure 2) is built on an inverted microscope and allows the precise positioning of a microcapillary, which is connected to a high-precision pump capable of aspirating and dispensing liquid in the subnanoliter range. The microcapillary is used to aspirate sample from a stock solution, to lyse individual eukaryotic cells, 

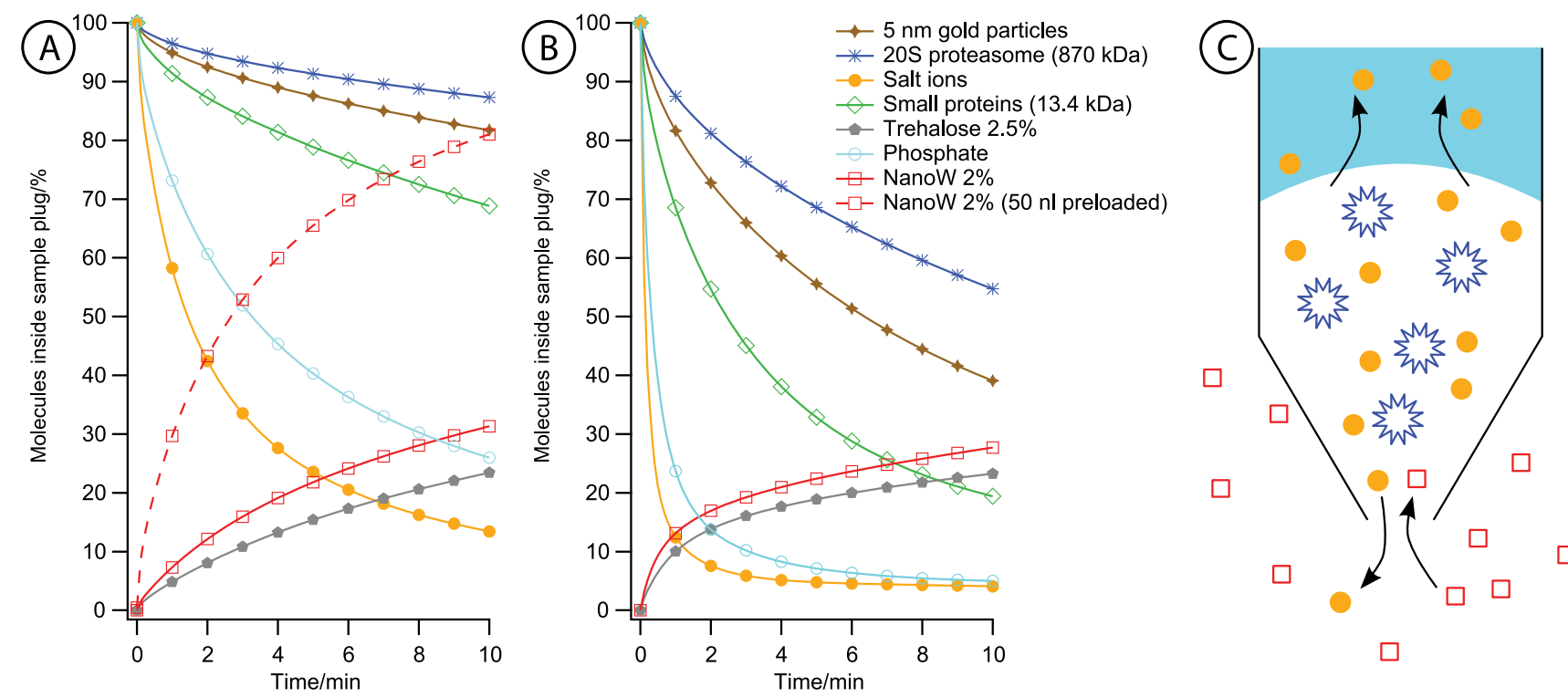

Figure 3. FEM simulation of the 3D diffusion process in the microcapillary and at its tip for two different geometries: (A) $100 \mu \mathrm{m}$ inner diameter (ID) and $30 \mu \mathrm{m}$ tip orifice, and (B) $250 \mu \mathrm{m}$ ID and $40 \mu \mathrm{m}$ tip orifice. For each species, the number of molecules was integrated over the initial sample plug volume of $4.5 \mathrm{~nL}$ and normalized by its initial number of molecules. Diffusion was allowed to occur between the sample tip and the stain (NanoW or trehalose) reservoir as well as between the sample plug and the system liquid $\left(\mathrm{ddH}_{2} \mathrm{O}\right)$ above it in the microcapillary. The dashed line represents the case for shorter conditioning times, where system liquid also contains negative stain $(2 \% \mathrm{NanoW})$, which is suited for certain low-salt-containing buffers. Diffusion coefficients can be extracted from Table 2. (C) Illustration of the diffusion process at the two interfaces.

and to dispense conditioned sample onto the EM grid i.e., all processing is performed in the same capillary without significant sample transport.

\section{THEORETICAL BACKGROUND AND EXPERIMENTAL CONSIDERATIONS}

Sample conditioning must ensure three crucial issues: First, sample salts must be removed; second, heavy metal salts (in the case of negative staining) must be introduced; and, third, the formation of insoluble precipitates between sample salts and the heavy metal ions must be prevented.

The conditioning process depends on the diffusion constants of the molecules and particles present, the viscosity of the liquids, and, last but not least, the geometry of the microcapillary tip. Finite element method (FEM) simulations were used to model the diffusive exchange of salt and negative stain ions between the sample plug and its environment (Figure 3A, B). Note that the diffusion process occurs at two interfaces: (i) at the capillary tip toward the conditioning reservoir and (ii) between the sampleplug and the system liquid ( $\mathrm{ddH}_{2} \mathrm{O}$ or negative stain) above it in the microcapillary (Figure $3 \mathrm{C}$ ). Because the area of the latter interface is larger, the diffusion process toward the system liquid is faster. The use of capillaries with larger inner diameters not only accelerates the conditioning process but also minimizes the glass surface the sample is exposed to (assuming that the volume is the same) and, therefore, avoids excessive sample loss by unspecific binding to the capillary walls.

Besides microcapillary geometry, the conditioning time also depends on sufficient desalting of the sample volume as well as on reaching a good negative stain or trehalose concentration inside the sample plug. Samples with a higher salt concentration need to be immersed for a longer period of time, because if the residual concentration of salt molecules is too high, salt crystals will form on the EM grid upon drying, as shown in Supporting Information S1. Fine-tuning is also required when the sample buffer, e.g., phosphate-buffered saline (PBS), forms insoluble precipitates with the heavy metal ions of the stain. Thus, the conditioning time is highly buffer dependent. The conditioning time can be significantly reduced by the use of buffers that, unlike PBS, do not form insoluble precipitates with the heavy metal salts of the negative stain. In this case, the system liquid can also contain negative stain solution for a faster increase of negative stain concentration inside the sample volume. Furthermore, conditioning time can be further reduced by using a low-salt buffer (see Application Note 1 section).

In order to validate the FEM simulations, we prepared EM grids with solutions of $5 \mathrm{~nm}$ nanogold beads (supplied by Cell Microscopy Center, Department of Cell Biology, University Medical Center Utrecht, The Netherlands), with and without conditioning. The nanogold particles were diluted in $50 \mathrm{mM}$ ammonium bicarbonate buffer. The same buffer was used for conditioning and as system liquid. The results shown in Supporting Information S2 demonstrate that the relative loss of particles is in good agreement with our simulations presented in Figure 3.

The graph in Figure 3 also reveals the effect of different diffusion coefficients on the amount of diffusive exchange. Due to the large difference (several orders of magnitude) between the diffusion coefficients of salt ions and larger proteins with masses ranging from few tens to thousands of $\mathrm{kDa}$, sample loss via diffusion can be neglected. Nevertheless, small protein molecules ( $<10 \mathrm{kDa}$ molecular weight or $<5 \mathrm{~nm}$ in diameter) will be lost with time (Figure 3). Although these molecules are below the size usually analyzed by EM, they might be potential binding partners of large protein complexes. If this is the case, we propose the use of one or both of the following measures: (i) Chemical fixation of protein complexes before sample preparation ${ }^{17}$ or (ii) reduction of the conditioning time by the use of lower ionic strength buffer systems and/or buffers that do not form insoluble precipitates with heavy metals, such as Tris (Tris- 
(hydroxymethyl)-aminomethane) buffers; conditioning times and protein-loss due to diffusion can thus be kept at a minimum (see Figure 5). As the physics of the exchange process taking place by diffusion is well understood, use of the immersiondiffusion method does not preclude quantitative evaluation of subsequent TEM measurements. Calibration factors can be estimated, allowing the relative occurrence of particles to be corrected.

So far, sample conditioning was a poorly addressed problem by microfluidic technologies. Mainly classical $\mathrm{H}$-filters ${ }^{18}$ were used, less frequently microdialysis technology was employed. ${ }^{11}$ Both approaches are technologically more challenging to implement than the immersion and diffusion method proposed here. Furthermore, the sample is exposed to larger surface areas than in the presented method, leading to significant loss by unspecific adsorption of proteins to the wall. In the proposed method the sample does not need to be transported during conditioning, which makes it also suitable for complex and more viscous samples.

\section{APPLICATION NOTE 1: NEGATIVELY STAINED SINGLE PARTICLES}

Single particle preparations of tobacco mosaic virus (TMV) or $20 \mathrm{~S}$ proteasome (20SP) samples were suspended in a standard PBS buffer $\left(2.7 \mathrm{mM} \mathrm{KCl}, 1.5 \mathrm{mM} \mathrm{KH}_{2} \mathrm{PO}_{4}, 136.9 \mathrm{mM} \mathrm{NaCl}, 8.9\right.$ $\mathrm{mM} \mathrm{Na} \mathrm{HPO}_{4} \cdot 7 \mathrm{H}_{2} \mathrm{O}, \mathrm{pH} 7.4$ ), which represented a worst-case buffer for sample preparation by negative stain. Volumes of $5 \mathrm{~nL}$ of sample were loaded into the tip of a microcapillary and dipped into a reservoir of negative stain solution for several minutes, to allow diffusive exchange of negative stain and salt ions. To sufficiently remove the phosphate salts from the sample, immersion times of 6-7 min were required. After staining, the total $5 \mathrm{~nL}$ sample volume was deposited onto a glow-discharged EM grid. Three different negative stains (2\% NanoW, 2\% NanoVan, and $0.5 \%$ ammonium molybdate) were tested, and good staining quality was achieved (Figure 4). Compared to the commonly used uranyl acetate stain, which has a $\mathrm{pH}$ around 4.3, these stains have a more physiological $\mathrm{pH}$, ranging from $\mathrm{pH} 6.8$ to 8 . They were also advantageous because uranyl-based negative stains tend to cross-link biological samples in solution. ${ }^{11}$ The known characteristics of the different negative stains were reflected by the results obtained using our method, e.g., very light staining was achieved using NanoVan as expected.

The conditioning time was significantly reduced by the use of a low-salt Tris-buffer. Figure 5 shows the staining achieved when single particle preparations (mix of TMV and Escherichia coli phages $)$ in a low-salt Tris-buffer $(20 \mathrm{mM}$ Tris- $\mathrm{HCl} \mathrm{pH} 7.4$ with $50 \mathrm{mM} \mathrm{NaCl}$ ) were conditioned for $3 \mathrm{~min}$ in a $2 \%$ NanoW solution with additional $10 \mathrm{~nL}$ of $2 \% \mathrm{NanoW}$ loaded in the system liquid. This shorter incubation time not only allows higher throughput for automated sample preparation but also ensures that any loss of small proteins is considerably reduced (see discussion above).

\section{APPLICATION NOTE 2: SINGLE-CELL VISUAL PROTEOMICS}

An individual adherent mammalian HEK 293 cell was lysed using three voltage pulses $(3 \times 250 \mu \mathrm{s}, 20 \mathrm{~V})$ applied by a microcapillary electrode (MCE) positioned in PBS buffer 20 $\mu \mathrm{m}$ above the cell. Its lysate was immediately aspirated into the MCE tip in a $3 \mathrm{~nL}$ liquid volume of PBS. After immersion of the MCE tip into a reservoir of $2 \%$ NanoW, $\mathrm{pH} 6.8$ for $10 \mathrm{~min}$, a total

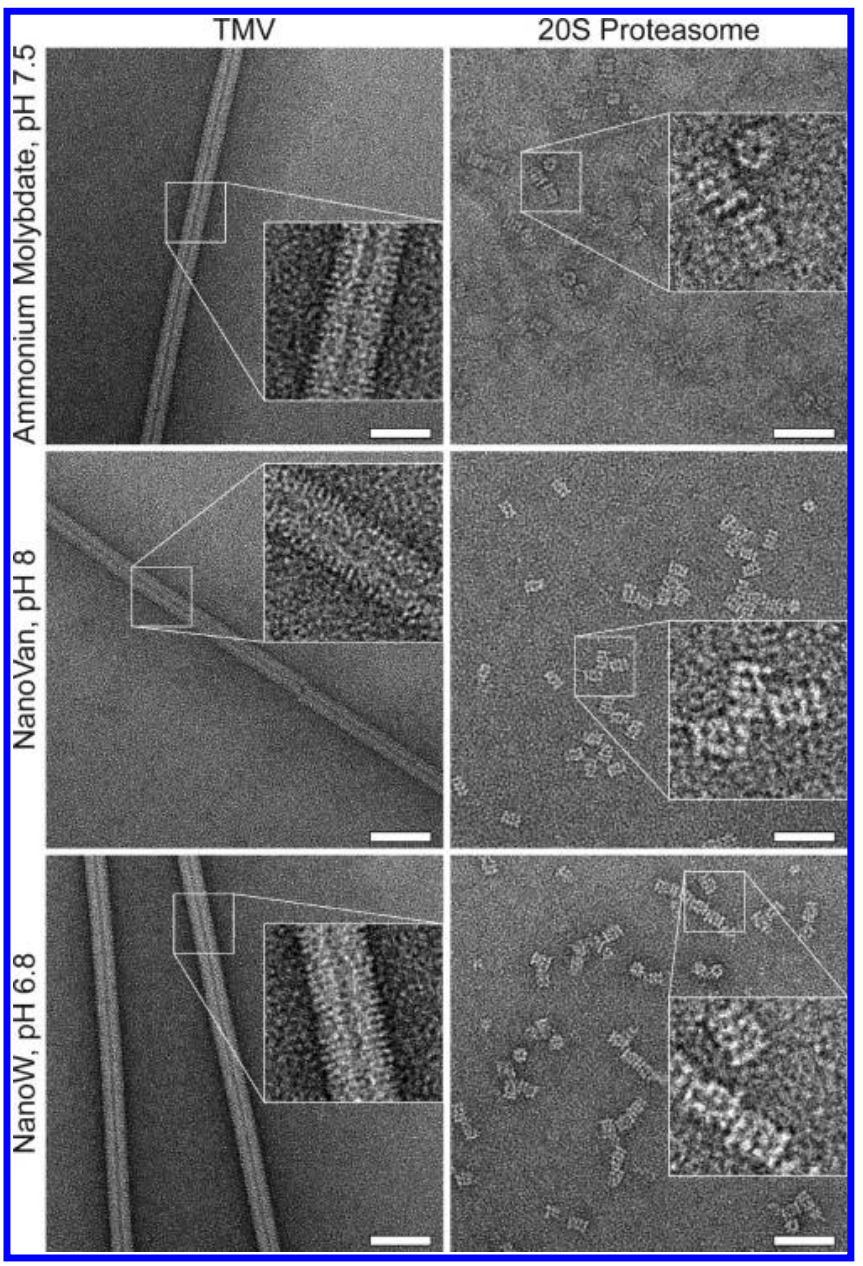

Figure 4. Single particles negatively stained by immersion-diffusion sample conditioning of samples solved in PBS buffer. Note that PBS buffer forms insoluble aggregates with heavy metal salts; therefore, longer conditioning times are needed (compare with Figure 5). Test samples of TMV (left column) and $20 \mathrm{~S}$ proteasome (right column), both in PBS buffer, were prepared using the indicated negative stain solutions by condition for $7 \mathrm{~min}$ in $2 \%$ solution (NanoW or NanoVan) or $\mathbf{0 . 5 \%}$ (ammonium molybdate). These stains differed in $\mathrm{pH}$ and staining characteristics. Note the lighter staining obtained with NanoVan at $\mathrm{pH} 8$. Scale bars represent $50 \mathrm{~nm}$.

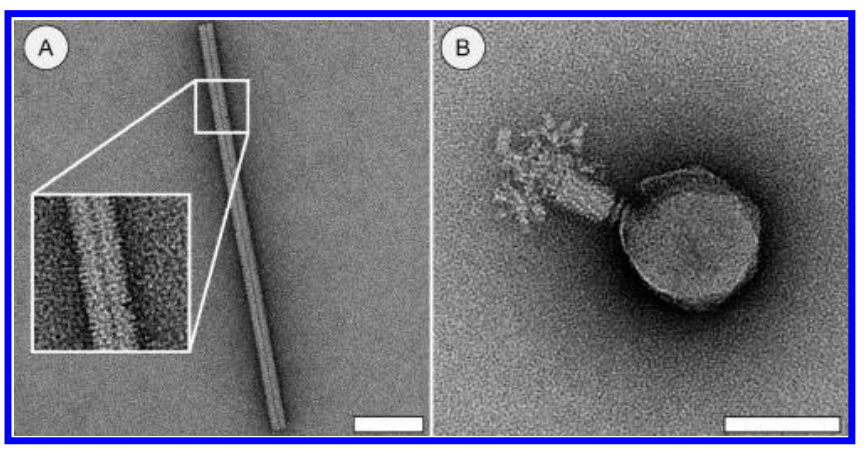

Figure 5. Single particles negatively stained by immersion-diffusion sample conditioning of samples solved in $20 \mathrm{mM}$ Tris-buffer. Scale bars represent $80 \mathrm{~nm}$. The inset in $A$ is magnified three times.

of $5 \mathrm{~nL}$ liquid was deposited from it onto a glow-discharged EM grid. The larger volume was required to dilute the highly concentrated lysate sample for EM. The high quality of the staining is documented by Figure 6. A filamentous structure 


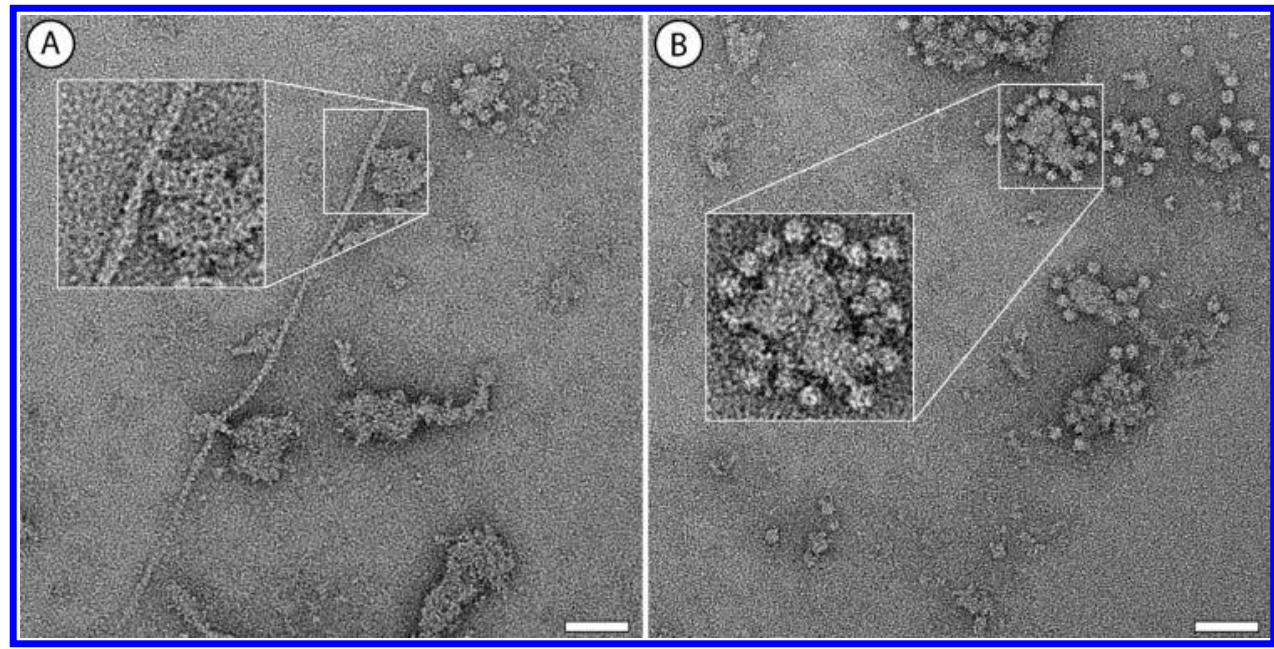

Figure 6. TEM image of negatively stained single-cell lysate from a HEK 293 cell. Prior to the cell lysis, the cell-culturing medium was exchanged with PBS buffer. (A) Filamentous structures resembling F-actin. (B) Membranes decorated with distinctive heads, resembling mitochondrial membranes with ATP-synthases. Negative stain: $2 \%$ NanoW, pH 6.8. Scale bars represent $50 \mathrm{~nm}$.

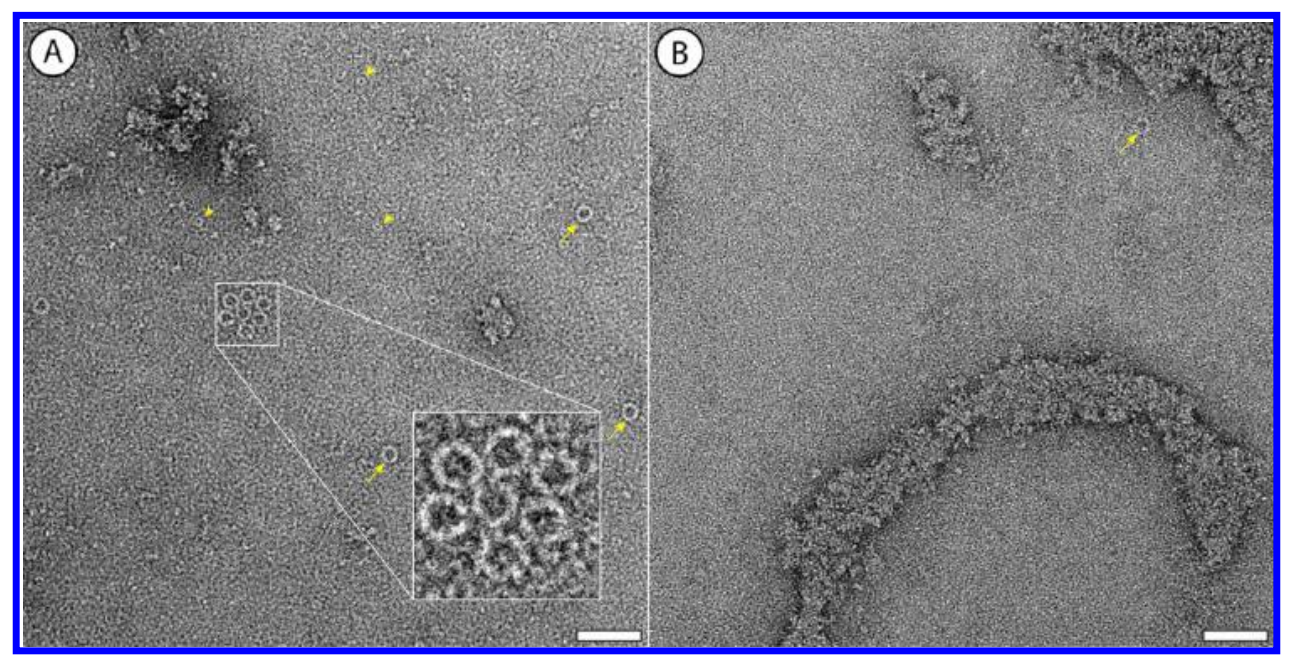

Figure 7. Effect of heat-shock on HEK 293 cells visualized by negative stain TEM of single-cell lysate in PBS buffer. (A) Cell lysate from a single HEK 293 cell after exposure to a $1 \mathrm{~h}$ heat-shock at $40{ }^{\circ} \mathrm{C}$. Multiple ring-shaped structures were found and were related to heat-shock proteins, such as Tric/CCT. Some of them are marked by arrows (large complex) and arrowheads (small complex). (B) Representative negative-control image of single-cell lysate from a cell without prior heat-shock. Only occasionally ring-like structures resembling heat-shock proteins are visible (arrow). Negative stain: 2\% NanoW. Scale bars represent $50 \mathrm{~nm}$.

resembling actin is evident in Figure 6A, and membranes decorated with distinctive protein heads are visible in Figure 6B. These membrane structures look like mitochondrial membranes with ATP-synthases. ${ }^{19}$

Figure 7A shows a representative image of the negatively stained single-cell lysate from a heat-shocked cell, and panel B shows the corresponding negative control. For the heat-shock, the HEK 293 cell culture was exposed to the elevated temperature of $40{ }^{\circ} \mathrm{C}$ for $1 \mathrm{~h}$, whereas the negative control lysate was from a cell cultured in parallel and treated identically except that it was always at the physiological temperature of 37 ${ }^{\circ} \mathrm{C}$. The frequency of ring-shaped structures was significantly increased in the single-cell lysate of the heat-shocked cell. Visually, these structures resemble negatively stained heat-shock proteins, e.g., the ring-like shape and the $13 \pm 0.7 \mathrm{~nm}$ diameter of the large rings (Figure 7A, arrows) are in good agreement with the reported structure of the Tric/CCT complex. ${ }^{20-22}$

\section{APPLICATION NOTE 3: EMBEDDING PROTEIN NANOCRYSTALS IN TREHALOSE FOR CRYO-EM}

Figure 8 shows an overview TEM image and the diffraction pattern of a lysozyme nanocrystal. Lysozyme nanocrystals were grown by the sitting drop method in a 96-well crystallization plate. ${ }^{23} 5 \mathrm{~nL}$ of a suspension containing nanocrystals not recognizable in a light microscope were aspirated from a crystallization well and conditioned in a $2.5 \%(\mathrm{w} / \mathrm{v})$ trehalose solution for $6 \mathrm{~min}$ as described above, dispensed onto the EM grid, and air-dried. The grid was subsequently frozen by manual plunging into liquid nitrogen, mounted into a cryo-EM sample holder, and inspected at liquid nitrogen temperature in a TEM by electron imaging and electron diffraction studies. Diffraction patterns with discernible spots at resolutions beyond $1 \AA$ were recorded (Figure 8). In contrast, when the trehalose conditioning step was omitted, the grid was covered by large clumps of electron dense material (Supporting Information Figure S3). The examination of pure water samples conditioned and deposited on the EM grid in the same way as the nanocrystals 


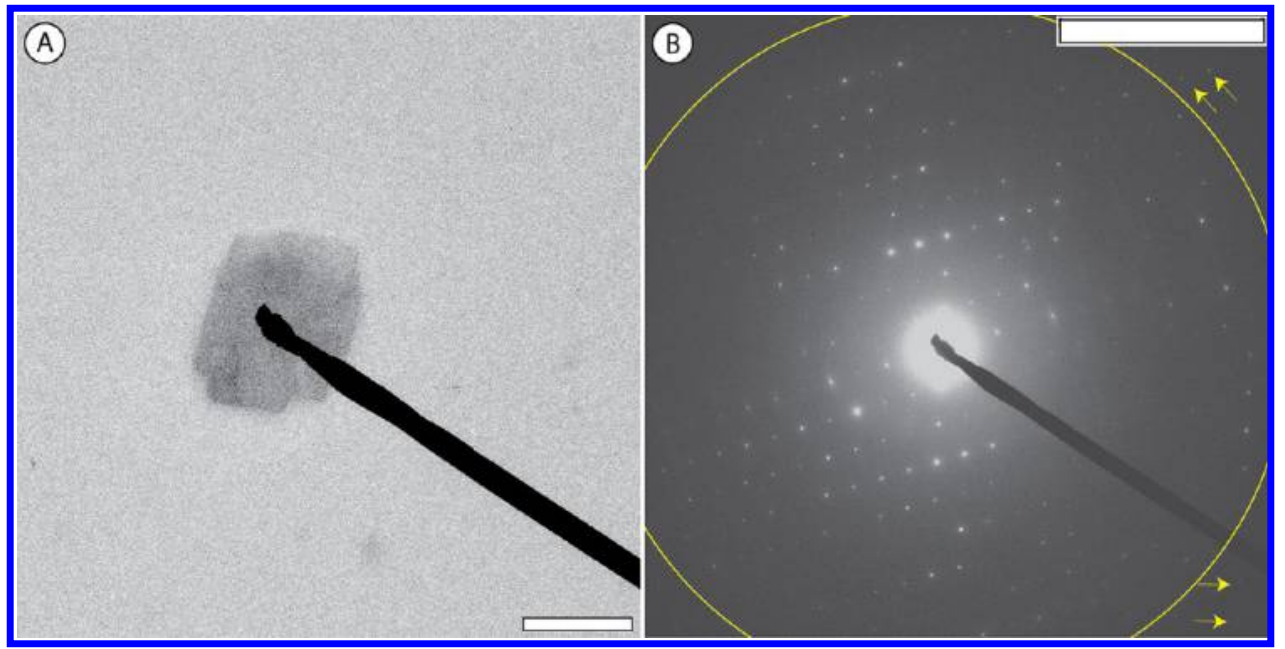

Figure 8. Nanocrystals conditioned in $2.5 \%(\mathrm{w} / \mathrm{v})$ trehalose for embedding. (A) Overview image showing a lysozyme protein crystal. (B) Electron diffraction of the nanocrystal depicted in (A). The yellow circle marks the resolution of $1 / 1 \AA$ A. Higher resolution diffraction spots are visible (yellow arrows). The scale bar represents $1 \mu \mathrm{m}$ in (A) and 1/1.7 $\AA$ in (B).

showed that trehalose was indeed present, forming a thin layer on the carbon/parlodion film covering the EM grid. Conditioning of the nanocrystals with pure water instead of trehalose, resulted in electron dense structures, however no diffraction was observed, suggesting that trehalose embedding and partial drying before freezing is a suitable method to preserve the structure of protein nanocrystals for EM investigations.

These results show that the conditioning method can be used for the preparation of 3D nanocrystals. This is not only of interest for investigations by electron diffraction and EM imaging but also for other analysis methods, such as serial crystallography achieved using synchrotron radiation or femtosecond exposures by X-ray free electron laser sources. ${ }^{24}$

\section{CONCLUSION}

We present a method for the conditioning and preparation of nanoliter sample volumes for subsequent analysis by EM. We show that the method can be used to prepare protein samples for single particle negative stain TEM (Figure 4) and to embed nanocrystals in trehalose for high-resolution analysis by electron diffraction (Figure 8). The minute amount and volume of material needed for sample preparation and the almost lossless procedure make it possible to study protein complexes of individual cells by a single-cell visual proteomics approach, as demonstrated by the heat-shock experiments (Figure 7).

\section{MATERIALS AND METHODS}

Instrument Setup. The instrument setup (Figure 2) is a further development of the setup described by Kemmerling and Arnold et al., ${ }^{16}$ which was initially built for single-cell lysis. It is mounted on an optical table (TMC Ametek, USA) and built around an inverted microscope (Zeiss Axiovert 200, Germany) with a motorized stage (Prior Scientific, UK) and a live-cell incubator (Tokai Hit, Japan). A microcapillary or, for single-cell lysis, a microcapillary electrode (MCE), is fixed to a $z$-axis stage (M-404.2PD, Physik Instrumente, Germany). The microcapillary tip is centered over the microscope objective. For single-cell lysis, the MCE surface is electrically connected to a $20 \times$ linear voltage amplifier (F20A, FLC Electronic AB, Sweden) and a function generator (33220A, Agilent, Switzerland) via a conductive sleeve. Cells are grown on a functionalized indium tin oxide (ITO) surface, which is grounded. The distal end of the microcapillary is connected to a high-precision syringe pump (Cetoni GmbH, Germany) and is filled with water (system liquid). Two surveillance cameras help to observe the experiments. All instruments are controlled via LabVIEW software. The openBEB software framework (www.openbeb.org), ${ }^{25}$ developed in house, is used to load each instrument program as an independent plug-in. Many experimental steps, e.g., cell lysis, sample loading, and sample deposition are now automatized using the macro language of openBEB.

Microcapillaries and Microcapillary Electrodes. Three types of fused silica microcapillaries (New Objective Inc., Woburn, MA, USA) were used. These had the same outer diameter (OD), but different inner diameters (ID) and tapered tips (Table 1). The outer surfaces of the

Table 1. Microcapillary Specifications and Application ${ }^{a}$

\begin{tabular}{cll}
$\begin{array}{c}\text { type, OD-ID- } \\
\text { TipD }(\mu \mathrm{m})\end{array}$ & $\begin{array}{c}\text { outer surface } \\
\text { (hydrophobic) }\end{array}$ & \multicolumn{1}{c}{ use } \\
FS360-250-40 & fused silica glass & $\begin{array}{c}\text { test samples/nanocrystals/Au } \\
\text { beads }\end{array}$ \\
FS360-250-40 & Pt & $\begin{array}{l}\text { single-cell analysis } \\
\text { low-salt test samples/ } \\
\text { nanocrystals/Au beads } \\
\text { FS360-100-30 }\end{array}$ \\
fused silica glass & Pt & single-cell heat-shock
\end{tabular}

${ }^{a}$ FS: fused silica; Pt: additional in house coating with a Ti/W sticky layer and $200 \mathrm{~nm} \mathrm{Pt}$; OD: outer diameter; ID: inner diameter; TipD: inner diameter at the tip; type: manufacturer's reference number.

glass tips were made hydrophobic by quickly flaming the tip, followed by immersion in a solution of sulfuric acid and polydimethylsiloxane (Regenabweiser, WSM Penzing). For electrical cell lysis, the outer surface of the microcapillaries was coated in house with $2 \mathrm{~nm}$ of Ti/W and $200 \mathrm{~nm}$ of Pt by sputter deposition and subsequently functionalized by immersion into a $1 \mathrm{M}$ ethanolic solution of 1-dodecanethiol for $24 \mathrm{~h}$ to increase the hydrophobicity.

Sample Conditioning by Immersion-Diffusion. The process of sample conditioning by immersion and diffusion is depicted in Figure 1. A few nanoliters of sample are aspirated from a lysed cell (Figure 1, A1) or from a sample stock delivered in a PCR tube (Figure 1, A2). Note that all capillaries are filled with system liquid (usually $\mathrm{ddH}_{2} \mathrm{O}$, in some cases also a $2 \%$ solution of negative stain ions in $\mathrm{ddH}_{2} \mathrm{O}$ ), which is in direct contact with the sample. The loaded microcapillary tip is immersed in a conditioning reservoir $(100 \mu \mathrm{L})$ of negative stain (NanoW 2\%, $\mathrm{pH} 6.8$, or NanoVan $2 \%, \mathrm{pH} 8$, both from Nanoprobes, USA, or ammonium molybdate $0.5 \%, \mathrm{pH} 7$, \#277908 from Sigma, Switzerland) or in a $2.5 \%$ $(\mathrm{w} / \mathrm{v})$ trehalose solution (TC-177, HiMedia Laboratories Pvt. Ltd., India) in $\mathrm{H}_{2} \mathrm{O}$ for 6 to $12 \mathrm{~min}$, mainly depending on the geometry of the microcapillary and the salt concentration in the sample. The transfer from the point of sample aspiration to the conditioning reservoir is automatized and only takes a few seconds. After conditioning via 
diffusive exchange of salt and negative stain ions or trehalose molecules (Figure 1B), the microcapillary is positioned above and close to a previously glow-discharged EM grid (200 mesh Cu grid, covered by a carbon-coated parlodion film). The sample is then dispensed onto the carbon surface and left to dry. A small spot that covers a few squares of the EM grid is formed (Figure 1C).

Diffusive Exchange Simulation. Diffusive exchange between the negative stain ions or trehalose, respectively, in the reservoir and the sample present in the microcapillary tip, as well as diffusive exchange between the sample and system liquid above it in the microcapillary, was simulated by the FEM in COMSOL Multiphysics 4.4. A 3D diffusion model was used with the transport of diluted species physics package. A simplified tapered microcapillary tip geometry, with the tip centered inside a cube of $8 \mu \mathrm{L}$ of negative stain or trehalose, was modeled. The negative stain or trehalose in the conditioning reservoir and the salt and protein in the sample plug were assigned typical concentrations and diffusion coefficients. The system liquid (double distilled water, $\mathrm{ddH}_{2} \mathrm{O}$, or negative stain) had a total volume of $50 \mathrm{~nL}$. The concentration was integrated over the volume of the sample plug $(4.5 \mathrm{~nL})$ on a time scale from 0 to $10 \mathrm{~min}$. The parameters used in the simulation are summarized in Table 2.

\section{Table 2. Simulation Parameters ${ }^{a}$}

\begin{tabular}{lccc}
\multicolumn{1}{c}{ solute } & $\begin{array}{c}\text { concentration } \\
(\mathrm{mM})\end{array}$ & $\begin{array}{c}\text { diffusion } \\
\text { coefficient }\left(\mathrm{m}^{2} / \mathrm{s}\right)\end{array}$ & ref \\
salt & 130 & $2 \times 10^{-9}$ & 26 \\
phosphate & 10 & $8.8 \times 10^{-10}$ & 27 \\
NanoW 2\% & 80 & $8.8 \times 10^{-10}$ & $\begin{array}{c}\text { Approximated } \\
\text { from } 27\end{array}$ \\
trehalose $2.5 \%$ & 66.1 & $5 \times 10^{-10}$ & 27 \\
$\begin{array}{c}\text { small proteins } \\
(13.4 \mathrm{kDa})\end{array}$ & 0.0746 & $1.14 \times 10^{-10}$ & 28 \\
$\begin{array}{c}\text { gold particles } \\
(5 \text { nm) }\end{array}$ & 0.00132 & $4.39 \times 10^{-11}$ & 29 \\
$\begin{array}{c}20 \text { S proteasome } \\
(870 \mathrm{kDa})\end{array}$ & 0.00115 & $2.28 \times 10^{-11}$ & 30
\end{tabular}

${ }^{a_{\text {Typical }}}$ concentrations were assigned to each solute. Diffusion coefficients were either obtained from literature, estimated by the Einstein-Stokes equation (gold particles), or approximated from known diffusion constants (tungstate and phosphate having similar hydration shells).

Single-Particle Test Samples. $20 \mathrm{~S}$ proteasome $(0.5 \mathrm{mg} / \mathrm{mL}$, 20SP, human, purified, BML-PW8720-0050, Enzo Life Sciences) and TMV (kindly supplied by Ruben Diaz-Avalos, New York Structural Biology Center, USA) were aspirated from $0.2 \mathrm{~mL}$ PCR tubes containing a few microliters of sample prepared in PBS buffer ( $\mathrm{pH}$ 7.4). A sample mixture containing TMV and Escherichia coli phages (vB_EcoM CBA120 from Petr Leiman, Laboratory of Structural Biology and Biophysics, EPFL) in $20 \mathrm{mM}$ Tris- $\mathrm{HCl}$ buffer ( $\mathrm{pH} 7.4$ ) with $50 \mathrm{mM} \mathrm{NaCl}$ served as the lowsalt-containing sample.

Cell Culture on Conducting Glass Slides. Adherent mammalian cells (HEK 293, Sigma, Switzerland) were cultured in polystyrene T25 flasks at $37{ }^{\circ} \mathrm{C}$ and $5 \%$ carbon dioxide with eagle's minimal essential medium (E-MEM, M2279, Sigma-Aldrich) supplemented with $2 \mathrm{mM}$ Glutamax (35050-038, Gibco), 1\% MEM nonessential amino acids (M7145, Sigma, Switzerland), and 10\% fetal bovine serum (10270-098, Gibco).

To split the cells, the medium was removed, and the flask was washed with $5 \mathrm{~mL}$ of $37^{\circ} \mathrm{C}$ warm PBS (Dulbecco's phosphate buffered saline; D8537, Sigma, Switzerland, $2.7 \mathrm{mM} \mathrm{KCl}, 1.5 \mathrm{mM} \mathrm{KH}_{2} \mathrm{PO}_{4}, 136.9 \mathrm{mM}$ $\left.\mathrm{NaCl}, 8.9 \mathrm{mM} \mathrm{Na}{ }_{2} \mathrm{HPO}_{4} \cdot 7 \mathrm{H}_{2} \mathrm{O}, \mathrm{pH} 7.4\right)$. To detach the cells, $1 \mathrm{~mL}$ of trypsin-EDTA solution (0.05\% trypsin, $0.53 \mathrm{mM}$ EDTA; 25300-054, Invitrogen, Switzerland) was added, and the cells were incubated at 37 ${ }^{\circ} \mathrm{C}$ for $3 \mathrm{~min}$. The detached cells were diluted with $5 \mathrm{~mL}$ of $37^{\circ} \mathrm{C}$ warm E-MEM and homogenized using a pipet. After centrifugation, the cells were resuspended in $5 \mathrm{~mL}$ of fresh, $37^{\circ} \mathrm{C}$ warm E-MEM for seeding.
For single-cell experiments, an aliquot of the cell suspension was seeded onto miniaturized Petri dishes on ITO-coated glass slides (Diamond Coatings UK, 8-12 $\Omega$ /square). The Petri dishes were produced as follows: ITO-coated glass slides were sonicated in detergent solution (1\% Alconox, Alconox Inc., USA) and ethanol for 10 min each, rinsed with $\mathrm{ddH}_{2} \mathrm{O}$, and dried in $\mathrm{N}_{2}$. Shortly before the cells were seeded onto the ITO slides, the slides were glow discharged and incubated with a 1:10 solution of poly-L-lysine in $\mathrm{H}_{2} \mathrm{O}$ (P8920, Sigma, Switzerland) for $5 \mathrm{~min}$. After a short wash with $\mathrm{ddH}_{2} \mathrm{O}$, the slides were dried at $60^{\circ} \mathrm{C}$ for $1 \mathrm{~h}$. Poly(dimethylsiloxane) (PDMS, Dow Corning SYLGARD 184) rings were then pressed onto the ITO surface to form sample wells, i.e., miniature Petri dishes, with a volume of about $300 \mu \mathrm{L}$. The PDMS wells were filled with cell culture medium $(300 \mu \mathrm{L})$, and around $50 \mu \mathrm{L}$ of HEK 293 cell suspension $\left(10^{4}\right.$ cells $)$ were added. The cultures were incubated for $1-2$ days at $37{ }^{\circ} \mathrm{C}$ and $5 \% \mathrm{CO}_{2}$. Prior to the single-cell lysis experiments, the cell culture medium was removed, and the cells were washed twice with $37^{\circ} \mathrm{C}$ warm PBS. The cells remained in PBS during the subsequent experiments.

Single-Cell Lysis. The ITO glass slide containing a sample well with live HEK 293 cells in PBS was placed in the slide holder on the inverted microscope of the cell lysis setup and put to electrical ground (Figure 2 A1). An individual cell was selected under the microscope, and the MCE tip was immersed into the buffer and placed $20 \mu \mathrm{m}$ above it. Three short voltage pulses $(3 \times 250 \mu \mathrm{s}$ at $16-20 \mathrm{~V})$ were delivered via the MCE to the targeted cell. The cell lysed within milliseconds, and its content was directly aspirated into the MCE tip in $3 \mathrm{~nL}$ of liquid by the syringe pump. For more details, see our previous work. ${ }^{16}$

Heat-Shock Experiments. An ITO-coated glass slide with HEK 293 cells in cell culture medium was inserted into the live-cell incubator stage (Figure 2). A temperature sensor was used to measure the temperature of the cell culture medium and to give feedback to the temperature controller. The cells were held at $40{ }^{\circ} \mathrm{C}$ for $1 \mathrm{~h}$ under a $5 \%$ $\mathrm{CO}_{2}$ atmosphere. The culture medium was then replaced by $37^{\circ} \mathrm{C}$ warm PBS buffer, and cell lysis was performed as described in the previous section. As a negative control, samples were prepared as described above from single cells from the same passage that were cultured in parallel without heat-shock, but otherwise under identical conditions.

Protein Nanocrystallization and Trehalose Embedding. A lysozyme crystallization trial was set up in a 96-well plate with sitting drop geometry. ${ }^{23}$ Wells were filled with $150 \mu \mathrm{L}$ of reservoir solution containing $0.6 \mathrm{M} \mathrm{NaCl}$ and $0.1 \mathrm{M}$ sodium acetate at three different $\mathrm{pHs}$. Lysozyme (A 3711.0010, BioChemica, Germany) was dissolved in $\mathrm{ddH}_{2} \mathrm{O}$ at a concentration of $50 \mathrm{mg} / \mathrm{mL}$. Sitting drops were composed of $2 \mu \mathrm{L}$ of lysozyme solution mixed with $2 \mu \mathrm{L}$ of reservoir solution.

After 7 days, $5 \mathrm{~nL}$ of crystallization solution were collected from a well ( $\mathrm{pH} 4.5$ ) that showed no visible crystals under the light microscope. The microcapillary tip containing this solution was then immersed in a reservoir of $2.5 \%(\mathrm{w} / \mathrm{v})$ trehalose for sugar embedding. After $6 \mathrm{~min}$, the microcapillary was raised, and the sample was directly deposited from its tip onto a glow discharged EM grid.

Electron Microscopy. Low-magnification overview images were acquired with a Philips CM10 (LaB6) TEM operated at $80 \mathrm{kV}$ and equipped with a $2 \mathrm{k}$ CCD camera (Olympus SIS, Münster, Germany). Higher magnification images were taken on a Philips CM200 (FEG) TEM operated at $200 \mathrm{kV}$ and equipped with a TVIPS F416 4k CMOS camera (TVIPS, Germany).

Electron diffraction was performed on the Philips CM200 TEM. The grid was cooled to liquid nitrogen temperature, placed in a Gatan-626 cryo-holder, inserted into the microscope, and imaged at cryo temperatures at around $-180{ }^{\circ} \mathrm{C}$. The camera length was $1 \mathrm{~m}$. The exposure time was $200 \mathrm{~ms}$, and the electron dose per recording was approximately $5 \mathrm{e}^{-} / \AA^{2}$.

\section{ASSOCIATED CONTENT}

\section{S Supporting Information}

The Supporting Information is available free of charge on the ACS Publications website at DOI: 10.1021/acsnano.6b01328.

S1 shows the desalting progress of single-cell lysate sample during conditioning. S2 shows experimental verification of 
the FEM simulations. S3 demonstrates the need for conditioning of $3 \mathrm{D}$ crystals prior to EM grid preparation (PDF)

\section{AUTHOR INFORMATION}

\section{Corresponding Author}

*E-mail: thomas.braun@unibas.ch.

\section{Notes}

The authors declare the following competing financial interest(s): The conditioning concept is part of the the patent application PCT/EP2015/065398.

\section{ACKNOWLEDGMENTS}

We thank the workshop of the Biozentrum of the University Basel for their support and S. Müller (C-CINA, Biozentrum, University of Basel) for carefully reading the manuscript. The project was supported by the Swiss Nanoscience Institute (SNI, projects P1201, P1305 and Nano Argovia SCeNA) and the Swiss National Foundation (SNF, project 200021_162521).

\section{REFERENCES}

(1) Li, X.; Mooney, P.; Zheng, S.; Booth, C. R.; Braunfeld, M. B.; Gubbens, S.; Agard, D. A.; Cheng, Y. Electron Counting and BeamInduced Motion Correction enable Near-Atomic-Resolution SingleParticle Cryo-EM. Nat. Methods 2013, 10, 584-590.

(2) Kuhlbrandt, W. Biochemistry. The Resolution Revolution. Science 2014, 343, 1443-4.

(3) Subramaniam, S.; Kuehlbrandt, W.; Henderson, R. CryoEM at IUCrJ: A New Era. IUCrI 2016, 3, 3-7.

(4) Scheres, S. H. W. J. RELION: Implementation of a Bayesian Approach to Cryo-EM Structure Determination. J. Struct. Biol. 2012, 180, 519-30.

(5) Grigorieff, N. J. FREALIGN: High-Resolution Refinement of Single Particle Structures. J. Struct. Biol. 2007, 157, 117-25.

(6) Rodriguez, J. A.; Ivanova, M. I.; Sawaya, M. R.; Cascio, D.; Reyes, F. E.; Shi, D.; Sangwan, S.; Guenther, E. L.; Johnson, L. M.; Zhang, M.; Jiang, L.; Arbing, M. A.; Nannenga, B. L.; Hattne, J.; Whitelegge, J.; Brewster, A. S.; Messerschmidt, M.; Boutet, S.; Sauter, N. K.; Gonen, T.; et al. Structure of the Toxic Core of $\alpha$-Synuclein from Invisible Crystals. Nature 2015, 525, 486-490.

(7) Nederlof, I.; Li, Y. W.; van Heel, M.; Abrahams, J. P. Imaging Protein Three-Dimensional Nanocrystals with Cryo-EM. Acta Crystallogr., Sect. D: Biol. Crustallogr. 2013, 69, 852-9.

(8) Goldie, K. N.; Abeyrathne, P.; Kebbel, F.; Chami, M.; Ringler, P.; Stahlberg, H. Methods in Molecular Biology; Electron Microscopy; Kuo, J., Ed.; Humana Press: Totowa, NJ, 2014, Vol. 1117, pp 325-341.

(9) Cheng, Y. A Primer to Single-Particle Cryo-Electron Microscopy. Cell 2015, 161, 450-457.

(10) De Carlo, S.; Harris, J. R. Negative Staining and Cryo-Negative Staining of Macromolecules and Viruses for TEM. Micron 2011, 42, 117-131.

(11) Kemmerling, S.; Ziegler, J.; Schweighauser, G.; Arnold, S. A.; Giss, D.; Mueller, S. A.; Ringler, P.; Goldie, K. N.; Goedecke, N.; Hierlemann, A.; Stahlberg, H.; Braun, T. Connecting $\mu$-Fluidics to Electron Microscopy. I. Struct. Biol. 2012, 177, 128-134.

(12) Coudray, N.; Hermann, G.; Caujolle-Bert, D.; Karathanou, A.; Erne-Brand, F.; Buessler, J. L.; Daum, P.; Plitzko, J. M.; Chami, M.; Mueller, U.; Kihl, H.; Urban, J. P.; Engel, A.; Rémigy, H. W. Automated Screening of 2D Crystallization Trials using Transmission Electron Microscopy: A High-Throughput Tool-Chain for Sample Preparation and Microscopic Analysis. I. Struct. Biol. 2011, 173, 365-374.

(13) Castro-Hartmann, P.; Heck, G.; Eltit, J. M.; Fawcett, P.; Samsó, M. The ArrayGrid: A Methodology for Applying Multiple Samples to a Single TEM Specimen Grid. Ultramicroscopy 2013, 135, 105-112.
(14) Mulligan, S.; Jain, T.; Duggan, E.; Liu, E.; Speir, J. A.; Cheng, A.; Nolan, J.; Carragher, B.; Potter, C. S. Typhon: Multiplexed TEM Sample Preparation. Microsc. Microanal. 2014, 20, 1158-1159.

(15) Chiu, P.-L.; Kelly, D. F.; Walz, T. The Use of Trehalose in the Preparation of Specimens for Molecular Electron Microscopy. Micron 2011, 42, 762-772.

(16) Kemmerling, S.; Arnold, S. A.; Bircher, B. A.; Sauter, N.; Escobedo, C.; Dernick, G.; Hierlemann, A.; Stahlberg, H.; Braun, T. J. Single-Cell Lysis for Visual Analysis by Electron Microscopy. J. Struct. Biol. 2013, 183, 467-473.

(17) Tang, X.; Bruce, J. E. Methods In Molecular Biology; Mass Spectrometry of Proteins and Peptides; Lipton, M. S., Paša-Tolic, L., Eds.; Humana Press: Totowa, NJ, 2009; Vol. 492.

(18) Yager, P.; Edwards, T.; Fu, E.; Helton, K.; Nelson, K.; Tam, M. R.; Weigl, B. H. Microfluidic Diagnostic Technologies for Global Public Health. Nature 2006, 442, 412-418.

(19) Walker, J. E.; Dickson, V. K. The Peripheral Stalk of the Mitochondrial ATP Synthase. Biochim. Biophys. Acta, Bioenerg. 2006, 1757, 286-296.

(20) Leitner, A.; Joachimiak, L. A.; Bracher, A.; Mönkemeyer, L.; Walzthoeni, T.; Chen, B.; Pechmann, S.; Holmes, S.; Cong, Y.; Ma, B.; Ludtke, S.; Chiu, W.; Hartl, F. U.; Aebersold, R.; Frydman, J. The Molecular Architecture of the Eukaryotic Chaperonin TRiC/CCT. Structure 2012, 20, 814-825.

(21) Schirmer, E. C.; Glover, J. R.; Singer, M. A.; Lindquist, S. HSP100/Clp Proteins: A Common Mechanism Explains Diverse Functions. Trends Biochem. Sci. 1996, 21, 289-296.

(22) Booth, C. R.; Meyer, A. S.; Cong, Y.; Topf, M.; Sali, A.; Ludtke, S. J.; Chiu, W.; Frydman, J. Mechanism of Lid Closure in the Eukaryotic Chaperonin TRiC/CCT. Nat. Struct. Mol. Biol. 2008, 15, 746-753.

(23) McPherson, A. Introduction to Protein Crystallization. Methods 2004, 34, 254-65.

(24) Hunter, M. S.; Segelke, B.; Messerschmidt, M.; Williams, G. J.; Zatsepin, N. A.; Barty, A.; Benner, W. H.; Carlson, D. B.; Coleman, M.; Graf, A.; Hau-Riege, S. P.; Pardini, T.; Seibert, M. M.; Evans, J.; Boutet, S.; Frank, M. Fixed-Target Protein Serial Microcrystallography with an X-Ray Free Electron Laser. Sci. Rep. 2014, 4, 6026.

(25) Ramakrishnan, C.; Bieri, A.; Sauter, N.; Roizard, S.; Ringler, P.; Müller, S. A.; Goldie, K. N.; Enimanev, K.; Stahlberg, H.; Rinn, B.; Braun, T. OpenBEB: Open Biological Experiment Browser for Correlative Measurements. BMC Bioinf. 2014, 15, 84.

(26) Bruus, H. Theoretical Microfluidics; Oxford University Press: Oxford, 2008.

(27) Stewart, P. S. Diffusion in Biofilms. I. Bacteriol. 2003, 185, 14851491.

(28) Young, M. E.; Carroad, P. A.; Bell, R. L. Estimation of Diffusion Coefficients of Proteins. Biotechnol. Bioeng. 1980, 22, 947-955.

(29) Einstein, A. Eine Neue Bestimmung der Moleküldimensionen. Ann. Phvs. 1905, 322, 549-560.

(30) Tanaka, K.; Yoshimura, T.; Kumatori, A.; Ichihara, A.; Ikai, A.j Nishigai, M.; Kameyama, K.; Takagi, T. Proteasomes (Multi-Protease Complexes) as $20 \mathrm{~S}$ Ring-Shaped Particles in a Variety of Eukaryotic Cells. I. Biol. Chem. 1988, 263, 16209-16217. 